INTERNATIONAL JOURNAL OF MULTidisciplinARY RESEARCH AND ANALYSis

ISSN(print): 2643-9840, ISSN(online): 2643-9875

Volume 04 Issue 04 April 2021

DOI: 10.47191/ijmra/v4-i4-21, Impact Factor: 6.072

Page No.- 493-495

\title{
Results of Surgical Treatment of Liver Echinococcosis in Our Practice
}

\author{
Omarov N.B. ${ }^{1}$, Aimagambetov M.Zh. ${ }^{2}$, Asylbekov E.M. ${ }^{3}$, Auyenov M.A. ${ }^{4}$, Abdrakhmanov S.T. ${ }^{5}$, \\ Mukash E.A. ${ }^{6}$, Akhmetzhanova D.0. ${ }^{7}$, Masalov A.E. ${ }^{8}$ \\ 1,2,3,45,6,7,8 Non-profit Joint Stock Company "Semey Medical University". Semey., Kazakhstan
}

\begin{abstract}
Results of surgical treatment of 45 patients with liver echinococcosis, operated on in the clinic of the University Hospital Non-profit joint-stock company "Semey Medical University" (UG NAO "MUS") from January 2017 to January 2021. these, there were 28 men (62.2\%), women - 17 (37.7\%). The average age was $47.6 \pm 3.2$ years.

Depending on the characteristics of the clinical situation, 2 variants of treatment tactics were performed for echinococcal cyst of the liver: in $37(82.2 \%)$ cases by the traditional method of echinococcectomy, in $8(17.8 \%)$ cases, pericystectomy. There were no lethal outcomes. All patients in satisfactory condition were discharged for outpatient treatment.
\end{abstract}

KEYWORDS: echinococcosis, diagnosis, treatment echinococcectomy, pericystectomy.

\section{INTRODUCTION}

Despite significant advances in the surgical treatment of liver echinococcosis, many of its aspects are far from definitive. Liver damage in echinococcosis ranges from 44 to $84 \%$ of cases among organ localization $[5,11]$.

Currently, the main method of treatment of liver echinococcosis remains surgical [3]. Today, the main disadvantages of surgical treatment are the high incidence of postoperative complications - up to $57 \%$, the formation of purulent and biliary fistulas - up to $31 \%$, recurrences of the disease - from 7 to $14 \%$, and deaths - up to $4.3 \%[1,8]$. As well as adverse cosmetic outcomes: long-term healing wounds, rough postoperative scars, fistulas, granulomas, postoperative hernias.

In the search for ways to improve the results of surgical treatment in recent years, such radical operations as liver resection and pericystectomy have been widely used. At the same time, many researchers point to the difficulty of performing radical surgery in the localization of cysts in the zones of the caval gates of the liver $[2,7,13]$. The main surgical intervention for liver echinococcosis is echinococcectomy $[9,10]$.

In this regard, the majority of surgeons in endemic regions perform open echinococcectomy with antiparasitic treatment of the residual cavity using one of the methods and various methods of eliminating the residual cavity [6, 14]. The currently available physical methods for treating the residual cavity do not provide a uniform effect over the area and depth, have the potential to damage the adjacent tubular structures of the liver and require specialized expensive equipment and cannot be used in most surgical departments.

The use of chemical compositions of alcohol, iodine, hypertonic solution and glycerin does not provide high-quality uniform treatment of the entire surface of the cyst, pockets, which is the reason for the recurrence of the disease [4].

In addition, today, in the case of a complicated or recurrent cyst, localization of the cyst in hard-to-reach parts of the liver, as well as with other contraindications to puncture treatment of cysts and laparoscopic echinococcectomy, or their ineffectiveness, there is no alternative method of minimally invasive treatment, which forces most surgeons to perform wide laparotomy [12].

Currently, all methods of surgery performed with liver echinococcosis have both advantages and disadvantages. Until now, in the literature, we have not come across proven advantages of this or that access for liver echinococcosis, depending on the peculiarities of the localization of the cyst. In addition, effective surgical treatment is hindered by a number of insufficiently studied aspects, problems of choosing an access and a method of operation, determining the nature and volume of an operation, a method of treating the cyst wall, the need for drainage or a method to eliminate the residual cavity, prevention and treatment of internal biliary fistulas.

Purpose of the study: To evaluate the results of echinococcectomy in patients with liver echinococcosis. 


\section{Results of Surgical Treatment of Liver Echinococcosis in Our Practice}

\section{MATERIALS AND METHODS}

Analyzed the results of surgical treatment of 45 patients with liver echinococcosis, operated in the clinic of the UG NAO "MUS" from January 2018 to January 2021. Of these, there were 28 men (62.2\%), women - 17 (37.7\%). The age of the patients varied from 18 to 69 years. The average age was $47.6 \pm 3.2$ years ( $M e=45 ; \mathrm{Q} 1=18 ; \mathrm{Q}=65$ ). The frequency of occurrence of echinococcosis liver cysts, as we found out, among the rural population is - in 31 (68.9\%), among the urban - in 14 ( $31.1 \%)$ patients.

Echinococcosis of the liver was established for the first time in $36(80 \%)$ patients out of 45 . With a relapse of the disease, 9 (20\%) patients were hospitalized. In 17 (37.8\%) patients, parasitic cysts were single, in $28(62.2 \%)$ - multiple. The overwhelming majority of patients included in this study were patients of working age, operated in a planned manner. Depending on the characteristics of the clinical situation, 2 variants of treatment tactics were performed for echinococcal cyst of the liver: in 37 $(82.2 \%)$ cases by the traditional method of echinococcectomy, in $8(17.8 \%)$ cases of pericystectomy.

Diagnosis of liver echinococcosis often included standard studies, and in some cases required an individual approach in terms of additional studies. All patients underwent complex clinical, laboratory and instrumental examinations of the abdominal organs, including ultrasound, computed tomography (CT), multispiral computed tomography (MSCT), and magnetic resonance imaging (MRI).

Intraoperative antiparasitic treatment of the residual cavity also plays a role. In our clinic (UG NAO "MUS") the following methods were used: $10 \% \mathrm{NaCl}$ solution ( $n=20,54.1 \%), 1 \%$ formalin solution $(n=7,29.7 \%), 5 \%$ povidone solution ( $n=10,27 \%$ ), with an exposure of 2 to 5 minutes.

\section{RESULTS}

The diagnosis was confirmed by serological (the detection of antibodies to echinococcus in the enzyme immunoassay is $86.6 \%$, eosinophilia was detected in $60 \%$ of the blood), according to ultrasound, the detection of echinococcosis is $95.5 \%$. According to the examination, cysts were found in all parts of the liver. Most often, echinococcal cysts were localized in the right lobe of the liver (Sg V, VI, VII, VIII): in 82.2\% of cases. In our study, three patients (6.7\%) had a relapse of hepatic echinococcosis.

Postoperative complications were observed in 4 patients: postoperative wound suppuration - 1 (2.2\%), subphrenic abscess - 1

(2.2\%), bile leakage - 2 (4.4\%). The average duration of a patient's stay in the hospital was 10.5 days, with a complicated course $-15.3+1.5 \mathrm{k} /$ days.

The frequency of occurrence among $68.8 \%$ of the rural population: Urdzhar district - 11 (35.5\%), Tarbagatai district - 1 (3.2\%), Abay district - 5 (16.1\%), Ayaguz district - 5 (16,1\%), Borodulikha district - 4 (12.9\%), Zaisan district - 1 (3.2\%), Kokpekty district - 2 (6.5\%), Kalbatau rural district - in 2 (6.5\%) patients ... There were no lethal outcomes. All patients in satisfactory condition were discharged for outpatient treatment.

After surgery, all the patients under observation were prescribed antiparasitic therapy with albendazole in order to prevent the recurrence of the disease. Three 28-day courses of antiparasitic therapy were used with a break of 14 days. Dispensary observation was carried out for three years. The criterion for removing patients from dispensary registration was the absence of clinical signs of echinococcosis, confirmed by instrumental and immunological examination methods.

\section{CONCLUSIONS}

Thus, echinococcosis of the liver is a common disease in the East Kazakhstan region with a predominant registration among the district residents - Urdzhar 35.5\% of the East Kazakhstan region. After intraoperative antiparasitic treatment of the residual cavity with povidone solution, in our study, in three patients $(6.7 \%)$, a relapse of hepatic echinococcus was noted. Patients with complicated liver echinococcosis should be operated on in a specialized institution with modern diagnostic and therapeutic equipment, highly qualified specialists.

\section{REFERENCES}

1) Gulov M.K., Kalmykov E.L., Zardakov S.M., Mukhabbatov D.K., Sadriev O.N. Echinococcosis of the liver: the role of computed tomography and morphological diagnostics of the state of liver tissue. Russian medical and biological bulletin named after Academician I.P. Pavlova. 2016; 24 (4): 104-11.

2) Clinical and epidemiological characteristics of echinococcosis in the Zaporozhye region / E.V. Ryabokon, O. V. Zarudnaya, N.S. Ushenina et al. // Zaporozhye medical journal. - 2013. - No. 3. - P. 63-65.

3) Complications of multiple echinococcosis of the liver / B. Monkhtoo, Ts. Ishdorzh, S. Erdene // Bulletin of the All-Russian Scientific Center of the Siberian Branch of the Russian Academy of Medical Sciences. - 2005. - No. 7 (45). - S. 78-80. 


\section{Results of Surgical Treatment of Liver Echinococcosis in Our Practice}

4) Rakhmatullaev A.R., Artykov K.P., Rakhmatullaev R.R. Laparoscopic simultaneous operations for combined surgical diseases of the abdominal organs. Avicenna Bulletin. 2016; 1: 21-3

5) Tolstokorov, A.S. Surgical tactics in patients with liver echinococcosis / A.S. Tolstokorov, Yu.S. Gergenreter // Saratov Journal of Medical Scientific Research. - 2009. - T. 5, No. 4. - P. 626-629.

6) Tursynbaev N.N. Long-term results of surgical treatment of patients with combined echinococcosis // Clinical medicine of Kazakhstan. -2010. - 2. - P.97-99.

7) Echinococcosis: current state of the problem / PS. Vetshev, G.Kh. Musaev, Magnetic resonance imaging in the complex diagnosis of hydatidant liver echinococcosis / E.S. Belysheva, V.G. Bychenko, A.N. Lotov et al. // Medical imaging. - 2003. - No. 2. - P. 6-12.

8) Alvarez Rojas CA, Romig T, Lightowlers MW. Echinococcus granulosussensu lato genotypes infecting humans - review of current knowledge. Int J Parasitol 2014; 44: 9-18

9) Charalambous, G.K. Three Cases of Primary Hydatidosis of the Gluteus Muscle: Our Experience in Clinical, Diagnostic and Treatment Aspects / G.K. Charalambous, V. A. Katergiannakis, A.J. Manouras // Chirurgia. - 2014. - Vol. 109, No. 4. - P. 555-558.

10) Kaltenbach TE, Graeter T, Mason RA, et al. Determination of vitality of liver lesions by alveolar echinococcosis. Comparison of parametric contrast enhanced ultrasound (SonoVue) with quantified 18F-FDG-PET-CT. Nuklearmedizin. 2015; 54: 43-9.

11) Madaminov E.M. Rezul'tat maloinvazivnogo varianta ekhinokokkektomii pecheni [The result of a minimally invasive variant of liver echinococcectomy]. Nauka, novye tekhnologii i innovatsii. 2015; 1: 111-2.

12) Multivisceral Echinococcosis: Concept, Diagnosis, Management / C. Grozavu, M. Ilias, D. Pantile // Chirurgia. - 2014. Vol. 109, No. 6. - P. 758-768.

13) Nakao M, Yanagida T, Konyaev S, Lavikainen A, Odnokurtsev VA, Zaikov VA, et al. Mitochondrial phylogeny of the genus Echinococcus (Cestoda: Taeniidae) with emphasis on relationships among Echinococcus canadensisgenotypes. Parasitology 2013; 140: 1625-36.

14) Rinaldi F, Brunetti E, Neumayr A, Maestri M, Goblirsch S, Tamarozzi F. Cystic echinococcosis of the liver: A primer for hepatologists. World J Hepatol 2014; 6 (5): 293-305 